\title{
Chemoradiotherapy with FOLFOX for esophageal squamous cell cancer with synchronous rectal cancer: Four case reports and a literature review
}

\author{
TAKAKO YOSHII $^{1}$, HIROKI HARA ${ }^{1}$, MASAKO ASAYAMA ${ }^{1}$, YOSUKE KUMEKAWA ${ }^{1}$, \\ SHOICHI MIYAZAWA ${ }^{1}$, NAOKI TAKAHASHI ${ }^{1}$, TOMOHIRO MATSUSHIMA ${ }^{1}$, \\ SATOSHI SHIMIZU $^{1}$ and YOSHIHIRO SAITO ${ }^{2}$
}

Departments of ${ }^{1}$ Gastroenterology and ${ }^{2}$ Radiotherapy, Saitama Cancer Center, Saitama 362-0806, Japan

Received January 29, 2019; Accepted July 16, 2019

DOI: $10.3892 / \mathrm{mco} .2019 .1945$

\begin{abstract}
Chemoradiotherapy (CRT) is a valuable treatment option for localized esophageal cancer. Conventional baseline chemotherapy for this type of cancer includes cisplatin and fluorouracil. Recently, CRT with leucovorinfluorouracil-oxaliplatin (FOLFOX) has become popular due to its convenience and lower toxicity. In Japan, the use of oxaliplatin for esophageal cancer is not yet approved, so experience with this treatment is limited to cases with colorectal cancer. As such patients are not usually included in clinical trials, little is known on the efficacy and safety of this treatment for this patient subpopulation, and treatment generalization in Japan is not allowed. We herein share our experience with CRT and FOLFOX for cases with esophageal cancer and synchronous rectal cancer at our institution. The clinical data of 4 patients who were treated for esophageal cancer with CRT/FOLFOX at our hospital between 2007 and 2016, who also had synchronous rectal cancer, were retrieved and analyzed. All the patients were male and had esophageal squamous cell cancer and synchronous rectal cancer. The median patient age was 68 years (range, 65-77 years). One patient received neoadjuvant CRT followed by surgery, and the other 3 patients received definitive CRT for esophageal cancer. FOLFOX was administered biweekly during radiotherapy (41.4-60 Gy). All 4 patients completed the treatment schedule and responded to CRT. No patients experienced progression of rectal cancer during treatment. Notably, 1 patient also achieved a complete response (CR) of rectal cancer after CRT for esophageal cancer. Moreover, 2 patients without dysphagia were treated as outpatients and achieved a CR. Encephalopathy was the only
\end{abstract}

Correspondence to: Dr Takako Yoshii, Department of Gastroenterology, Saitama Cancer Center, Komuro 780, Ina, Kitaadachi-gun, Saitama 362-0806, Japan

E-mail: takako_y@cancer-c.pref.saitama.jp

Key words: esophageal cancer, chemoradiotherapy, FOLFOX, rectal cancer, double cancer reported grade 3 adverse event. Although the present study included a limited number of cases, the findings suggest that CRT with FOLFOX may be a valuable option for the treatment of patients with esophageal squamous cell cancer and synchronous rectal cancer.

\section{Introduction}

Esophageal cancer (EC) is the ninth most common type of cancer and the sixth most common cause of cancer-related mortality globally (1). EC is associated with considerable morbidity and carries a poor prognosis in its later stages. This disease requires a multidisciplinary approach, such as neoadjuvant chemotherapy or chemoradiotherapy (CRT) plus surgery for locally advanced disease. Definitive CRT is also recognized as a curative treatment option (1-3), particularly in patients who are not surgical candidates.

A number of clinical studies of radical CRT for EC have been conducted since the 1980s, and radiotherapy (50-60 Gy) with cisplatin and fluorouracil (CF) has become the standard of care $(4,5)$. However, $\mathrm{CF}$ is associated with a number of problems. It has been reported that local failure and toxicity-related deaths/other life-threatening toxicities were observed in 46 and $20 \%$ of patients with EC, respectively, who receive this CRT, and that $41 \%$ patients with EC could not complete the CRT as planned (6). CRT with CF may also cause thrombosis, sudden death, or other toxicities, and it requires hospital admission due to the requirement for prolonged intravenous hydration for cisplatin, and 5 days continuous infusion for $5 \mathrm{FU}$.

Therefore, a safer and more useful regimen of CRT for EC is urgently needed. A phase II/III clinical study (PRODIGE5/ACCORD17) was conducted to investigate the superiority of leucovorin-fluorouracil-oxaliplatin (FOLFOX) to $\mathrm{CF}$ as the chemotherapy component of CRT, replacing cisplatin with oxaliplatin, which rarely induces kidney toxicity and does not require intravenous hydration. Although the results of the study did not meet the endpoint hypothesized, namely that FOLFOX is superior to $\mathrm{CF}$, the researchers demonstrated its lower toxicity and non-inferior survival to $\mathrm{CF}$; thus, CRT with FOLFOX for EC is recognized as one of the standard treatment options in Europe (7-9). 
In Japan, oxaliplatin along with several other drugs have not yet been approved for EC treatment, and CF is commonly used as the chemotherapeutic component of CRT (10-12). FOLFOX has been approved only for patients with colorectal or gastric cancer. Thus, clinical data on the feasibility of this treatment for EC in Japan are rare. We herein report the cases of 4 patients with esophageal squamous cell cancer (ESCC) and rectal cancer (RC) who were treated with CRT with FOLFOX in our hospital.

\section{Patients and methods}

Patients. Between January 2007 and December 2016, 740 patients were registered in our hospital's database of chemotherapy for EC; that database was searched for patients who received CRT with FOLFOX, and their clinical data were investigated (Table I).

Treatment. FOLFOX was administered every 2 weeks for 3-6 cycles, with the first 3 cycles administered concurrently with radiotherapy. During each cycle, oxaliplatin $\left(85 \mathrm{mg} / \mathrm{m}^{2}\right)$ was administered as a $2 \mathrm{~h}$ intravenous infusion in $250-500 \mathrm{ml}$ of $5 \%$ glucose on day 1 , concurrently with leucovorin $\left(200 \mathrm{mg} / \mathrm{m}^{2}\right)$ as a $2 \mathrm{~h}$ intravenous infusion. Fluorouracil $\left(400 \mathrm{mg} / \mathrm{m}^{2}\right)$ was administered as a $10 \mathrm{~min}$ intravenous bolus dose on day 1 , followed by continuous intravenous infusion of fluorouracil $\left(1,600 \mathrm{mg} / \mathrm{m}^{2}\right)$ over 2 days, which was based on the PRODIGE5/ACCORD17 trial (8).

Radiation therapy was delivered with megavoltage equipment ( $>6 \mathrm{MV})$ with anterior/posterior opposed or multi-field irradiation and continuous bilateral oblique (off-cord) portals, except for neoadjuvant cases. The patients were treated 5 days per week at 1.8-2 Gy/day to a total dose of 41.4-60 Gy. The details of the regimen for each patient are provided in the individual case report descriptions and Table II.

Response to treatment. Response was assessed by esophageal endoscopy, and neck-to-abdomen computed tomography (CT) 2-3 weeks after the completion of radiotherapy or 6 cycles of FOLFOX for each case. The response of primary tumors of the esophagus was assessed based on the criteria of the Japanese Classification of Esophageal Cancer (11th edition) (13). According to these criteria, a CR required meeting all of the following: i) No evidence of tumor except flat erosion, white exudate or a scar, ii) a negative biopsy, iii) no new lesions and iv) confirmation of i-iii with at least a 4-week interval. Progressive disease (PD) required meeting any of the following criteria: i) Tumor growth and ii) appearance of any new lesions or metastasis. If neither the criteria of CR or PD were met, the response was categorized as non-CR/non-PD. Overall response was assessed bases on RECIST version 1.1. (14).

Toxicity was assessed according to Common Terminology Criteria for Adverse Events (CTCAE) version 4.0 (15) during the treatment course. The outcome and late toxicities were recorded until patient death or the final visit on the chart. Survivors were followed up by tri-monthly CT and endoscopy, and physical examination after the confirmation of CR.

The analysis was performed in April 2017. All patients provided written informed consent for treatment by CRT with FOLFOX. Publication of this retrospective analysis was approved by the Institutional Review Board of our hospital.

\section{Results}

Four patients with EC treated by CRT with FOLFOX were selected from the database. All patients had synchronous ESCC and $\mathrm{RC}$. The patient characteristics and clinical courses are summarized in Tables I and II. The details are described below.

Case 1. A 68-year-old male patient presented with dysphagia and was diagnosed with type 2 ESCC (Lt, cT3N1) and RC (Rs, cT4aN1), whereas liver metastases were synchronously diagnosed. Although liver biopsy was not performed for histological analysis, the liver metastases were clinically determined to be derived from the RC, as progression of its primary and lymph node sites was observed, with significant increases in the tumor marker carcinoembryonic antigen (CEA) level to $31.2 \mathrm{ng} / \mathrm{ml}$ (normal, $<5 \mathrm{ng} / \mathrm{ml}$ ). As the patient had synchronous stage IV metastatic RC, his ESCC did not fulfil the indications for radical, invasive surgery; therefore, CRT was performed. Due to the synchronous RC, FOLFOX was selected as the concurrent chemotherapy. Radiotherapy (60 Gy/30 Fr/46 days, omitting elective nodes due to the incurability of this condition) was performed with 3 cycles of FOLFOX, as described in Methods. In the following evaluation, although the response of the primary site was assessed as non-CR due to persistent severe esophagitis, the primary ESCC had regressed. The lymph nodes had also decreased in size. The overall response was assessed as non-CR/non-PD in accordance with RECIST. As regards RC, the outcome was determined as stable disease (Fig. 1).

Chemotherapy was continued focusing on the metastatic RC. Although FOLFOX was planned to continue for 6 cycles after completing radiotherapy based on the PRODIGE5/ACCORD17 trial, the subsequent treatment was changed to fluorouracil-leucovorin-irinotecan (FOLFIRI)-cetuximab, as the therapeutic efficacy of FOLFOX in $\mathrm{RC}$ was considered to be limited, and the RAS status of the $\mathrm{RC}$ was wild-type. The patient developed a fever $\left(38-39^{\circ} \mathrm{C}\right)$ 10 days after the initiation of FOLFIRI-cetuximab. As grade 3 neutropenia was also observed $(960 / \mu 1)$, the patient was hospitalized and treated with antibiotics. Although the neutrophil count had recovered to $10,910 \mu \mathrm{l}$ by day 12 , the patient had persistent fever and developed hypoxemia on day 19. Chest computed tomography was performed and revealed interstitial pneumonia due to cetuximab. The patient did not respond to pulse steroid therapy and succumbed to aggravated interstitial pneumonia 46 days after the start of FOLFIRI-cetuximab.

Case 2. A 68-year-old male patient presented with dysphagia and was diagnosed with type 2 ESCC (Mt, cT3N1) and RC (Rs, cT3N1). As both tumors were resectable, radical surgery was planned at the same time after neoadjuvant therapy for the ESCC. For the RC, the neoadjuvant therapy adopted was CRT with FOLFOX. As this was a planned surgery, the dose of radiotherapy was adjusted to $41.4 \mathrm{~Gy} / 23 \mathrm{Fr}$, which was generally administered as neoadjuvant CRT, with 3 cycles of FOLFOX administered concurrently. The response of the ESCC was evaluated as non-CR/non-PD, with slight regression of the 
Table I. Patient characteristics.

\begin{tabular}{lcccll}
\hline Case no. & Sex/age (years) & Histology & TNM (UICC-7th) & Setting & \multicolumn{1}{c}{$\begin{array}{c}\text { Multiple primary } \\
\text { cancers }\end{array}$} \\
\hline 1 & $\mathrm{M} / 68$ & SCC & T3/N1/M0 & dCRT & RC: cT4aN1H2 \\
2 & $\mathrm{M} / 68$ & SCC & T3/N1/M0 & Neo-CRT & RC: cT3N1M0 \\
3 & $\mathrm{M} / 65$ & SCC & T3/N2/M0 & Neo $\rightarrow$ dCRT & RC: cT3N0H0 \\
4 & M/77 & SCC & T2/N1/M0 & dCRT & RC: preceding \\
& & & & surgery (pStage IIIB)
\end{tabular}

M, male; F, female; SCC, squamous cell cancer; dCRT, definitive chemoradiotherapy; RC, rectal cancer; Neo-CRT, neoadjuvant chemoradiotherapy.
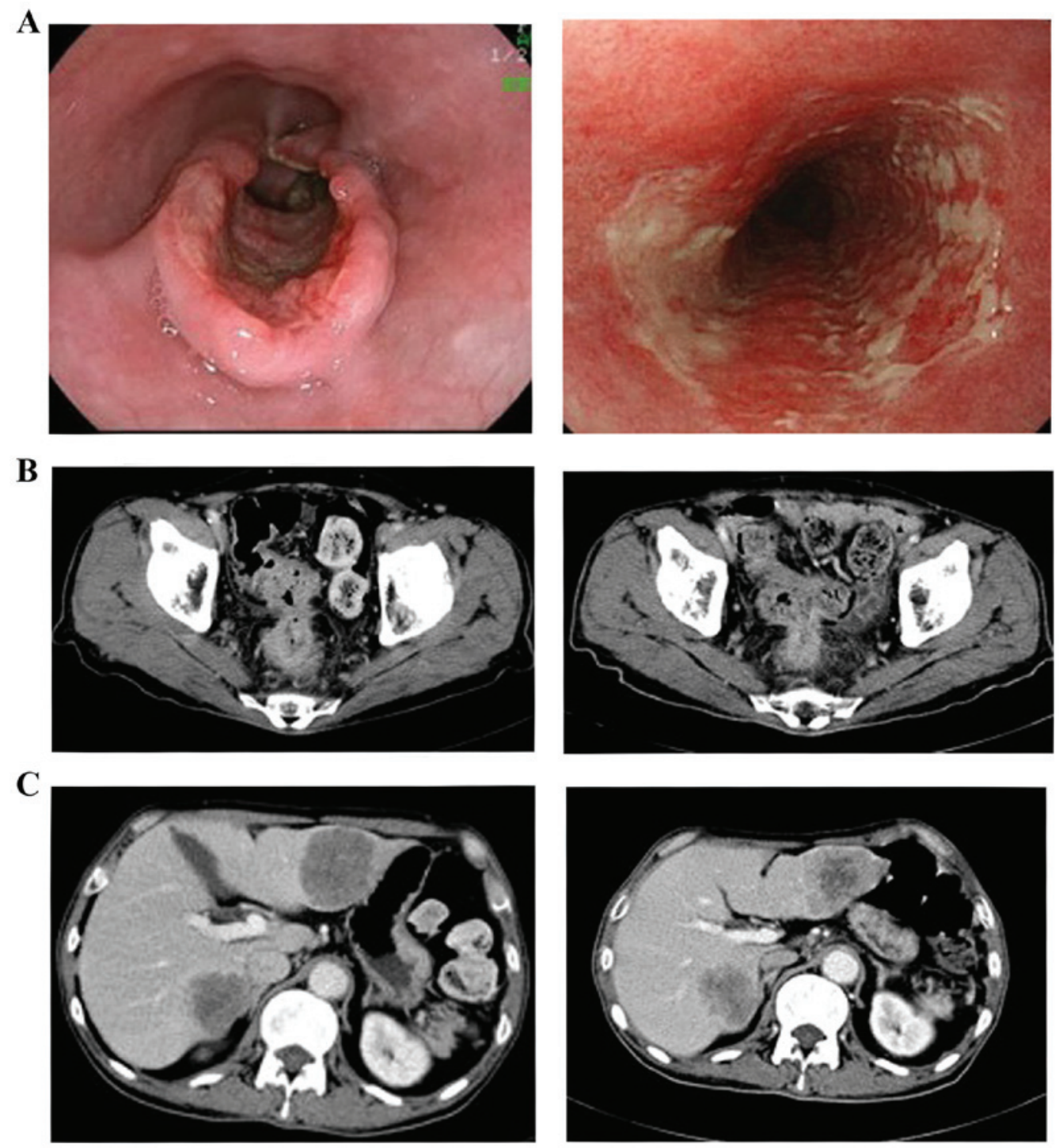

Figure 1. Case 1. (A) Esophageal cancer pre- and post-chemoradiotherapy. Despite the persistent esophagitis, the tumor has almost disappeared. (B) Rectal cancer pre- and post-chemoradiotherapy. (C) Liver metastases pre- and post-chemoradiotherapy.

lymph node metastases and tumor shrinkage, but prolonged esophagitis due to the radiation. For the $\mathrm{RC}$, the outcome was also determined to be non-CR/non-PD (Fig. 2). During the 3rd cycle of FOLFOX, the patient developed 5-FU-induced encephalitis with hyperammonemia $(252 \mu \mathrm{g} / \mathrm{dl})$. Although he rapidly recovered after administration of branched-chain amino acids, continuation of the remaining FOLFOX cycle, similar to PRODIGE5/ACCORD17, was considered to be harmful. At that time, both cancers were assessed as resectable, so simultaneous radical surgeries were performed. 

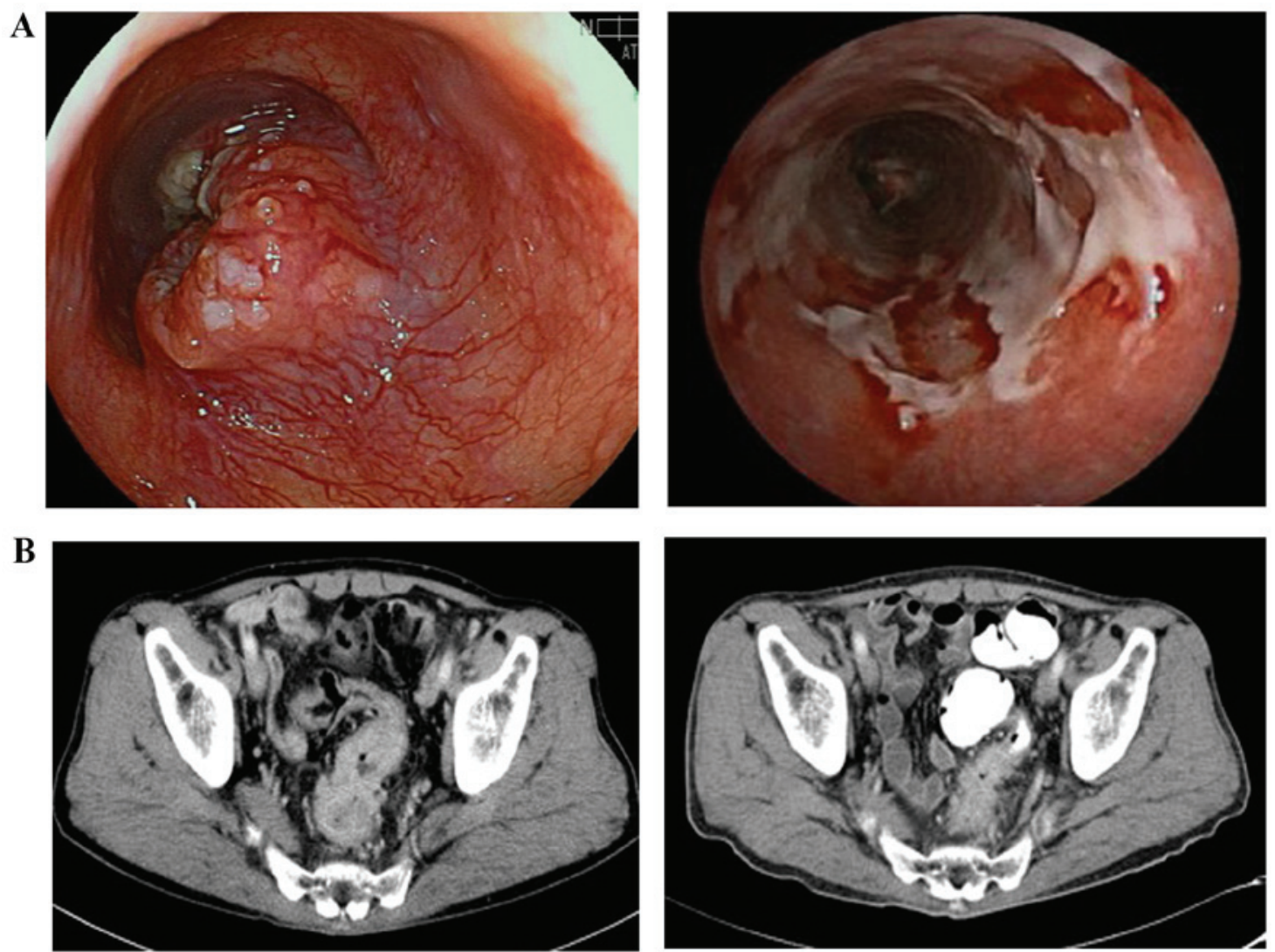

Figure 2. Case 2. (A) Esophageal cancer pre- and post-chemoradiotherapy. Despite the persistent esophagitis, the tumor has almost disappeared. (B) Rectal cancer pre- and post-chemoradiotherapy.
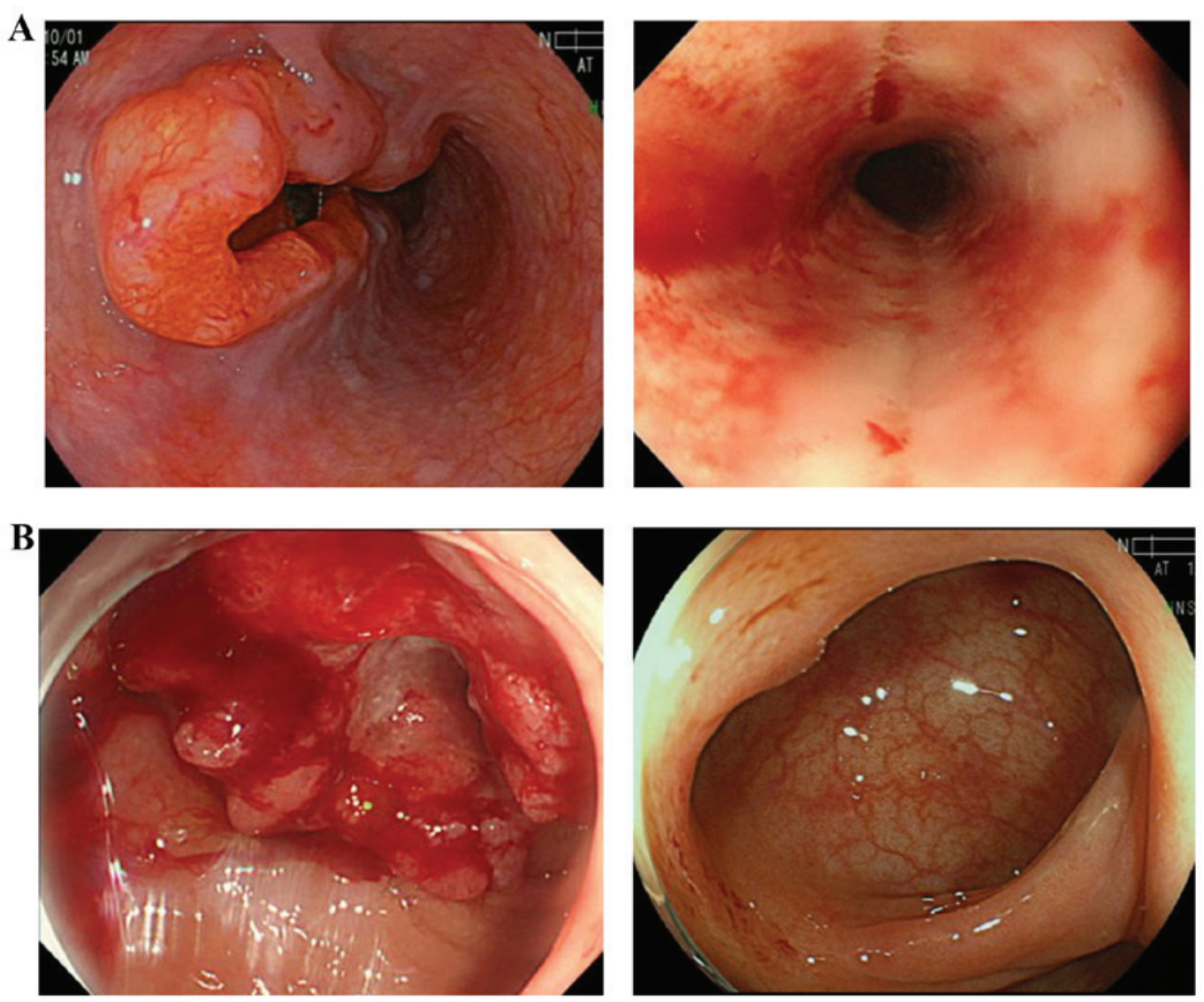

Figure 3. Case 3. (A) Esophageal cancer pre- and post-chemoradiotherapy. Despite the persistent esophagitis, the tumor has almost disappeared. (B) Rectal cancer pre- and post-chemoradiotherapy. The rectal cancer exhibits complete response. 
Table III. Adverse events ( $n=4)$.

\begin{tabular}{llc}
\hline & \multicolumn{2}{c}{ Grade, $\mathrm{n}(\%)$} \\
\cline { 2 - 3 } Adverse events $^{\mathrm{a}}$ & All & $\geq 3$ \\
\hline Hematological & & \\
Neutropenia & $3(75)$ & 0 \\
Anemia & $3(75)$ & 0 \\
Thrombocytopenia & $2(50)$ & 0 \\
Febrile neutropenia & 0 & 0 \\
Anorexia & $3(75)$ & 0 \\
Esophagitis & $3(75)$ & 0 \\
Constipation & $3(75)$ & 0 \\
Dysphagia & $3(75)$ & 0 \\
Non-hematological & & \\
Erythema & $2(50)$ & 0 \\
Diarrhea & $2(50)$ & 0 \\
Hypokalemia & $2(50)$ & 0 \\
Nausea & $2(50)$ & 0 \\
Paresthesia & $2(50)$ & 0 \\
Encephalopathy & $1(25)$ & $1(25)$ \\
Vomiting & $1(25)$ & 0 \\
Hiccups & $1(25)$ & 0 \\
Pneumonia & $1(25)$ & 0 \\
Mucositis & 0 & 0 \\
Thrombocytopenia & 0 & 0 \\
Renal insufficiency & 0 & 0 \\
& &
\end{tabular}

${ }^{\mathrm{a} C}$ Common Terminology Criteria for Adverse Events (version 4).

Unexpectedly, the resection of the ESCC was classified as R2. As the patient did not achieve recovery of his performance status, additional treatment for ESCC was not feasible, so close follow-up was planned. The patient developed recurrence of mediastinal lymph node metastasis 84 days after surgery. His performance status at the time was poor, and he succumbed to ESCC 112 days after surgery.

Case 3. A 65-year-old male patient presented with dysphagia and was diagnosed with type 2 ESCC (Mt, cT3N2) and RC (Rs, cT3N0). Due to mediastinal lymph node metastases (no. 112) invading the aorta, they were deemed to be unresectable. Therefore, the treatment selected for the ESCC was definitive CRT with FOLFOX, followed by radical surgery for the RC. Three cycles of FOLFOX were administered with definitive radiotherapy ( $60 \mathrm{~Gy} / 30 \mathrm{Fr} / 46$ days), followed by another 3 cycles of FOLFOX. The response of the ESCC was evaluated after all 6 cycles of chemotherapy as non-CR/non-PD, with regression of lymph node metastases and prolonged radiation esophagitis, but the primary tumor had shrank notably. On the other hand, the outcome of FOLFOX for RC was CR (Fig. 3). Considering a delayed CR of the ESCC, the patient was closely followed up to avoid invasive salvage surgery. To date, neither the ESCC nor the RC have progressed. The progression-free survival (PFS) for both cancers has been $>2$ years since CRT, without surgery. 


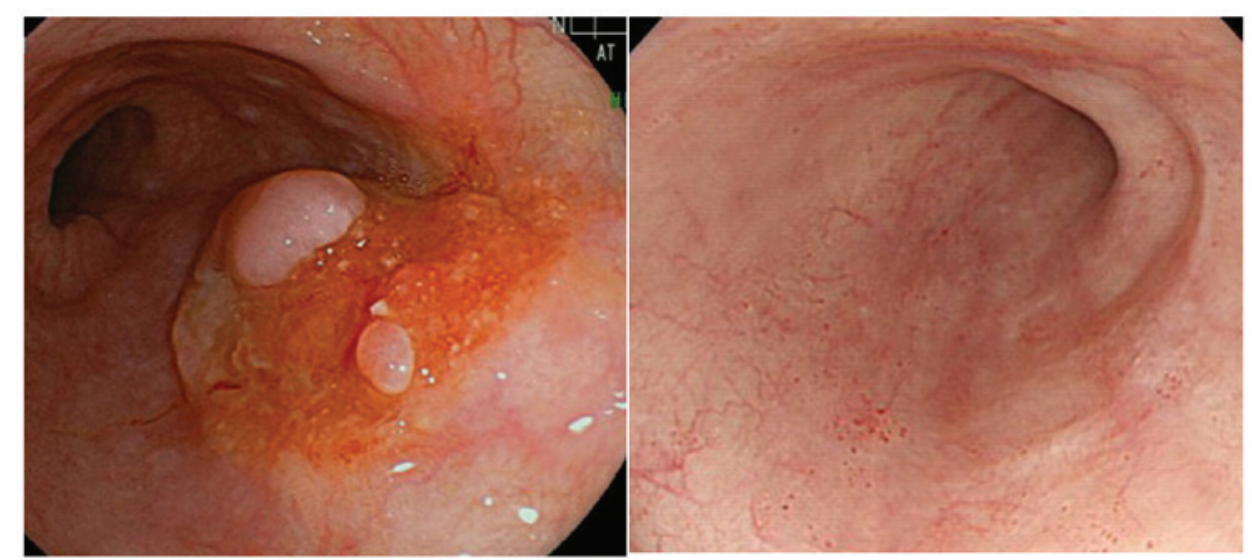

Figure 4. Case 4. Esophageal cancer pre- and post-chemoradiotherapy. The esophageal cancer exhibits complete response.

Case 4. A 77-year-old male patient presented with defecation problems. Type 2 circumferential RC (Rsa, cT3NO) and ESCC (Lt, cT2N1) were diagnosed. The RC caused severe stenosis, and there was a risk of ileus, so radical surgery was performed. Subsequently, it was decided to treat the ESCC with definitive CRT due to an anatomic anomaly (a right aortic arch) that would make the surgery for the ESCC difficult. As the pathological stage of the RC after resection was pStage IIIB (pT3N1), adjuvant chemotherapy with FOLFOX was scheduled for 6 months. Therefore, CRT with FOLFOX was selected for this ESCC case according to the PRODIGE5/ACCORD17 trial, as described in Methods. After completion of 6 cycles of FOLFOX with definitive radiation (60 Gy/30 fr/46 days), the patient developed grade 2 peripheral sensory neuropathy due to treatment with oxaliplatin. Therefore, his chemotherapy was switched to capecitabine monotherapy for the remaining 3 months of adjuvant chemotherapy for the RC. The outcome for the ESCC was determined as CR (Fig. 4). The patient has survived without progression for $>1$ year and 9 months since the completion of FOLFOX chemotherapy.

The encephalitis in patient 2 was the only grade $\geq 3$ adverse event observed among the 4 patients, whereas the other AEs were common events caused by CRT with CF (Table III).

\section{Discussion}

In the RTOG 85-01 study, CRT was found to be notably superior to radiotherapy alone in terms of survival prolongation for esophageal cancer. In that study, CF was concurrently administered $\left(1,000 \mathrm{mg} / \mathrm{m}^{2} 5\right.$-FU on days $1-4 ; 75 \mathrm{mg} / \mathrm{m}^{2}$ cisplatin every 28 days) (4). In the RTOG 94-05 study, which was a randomized controlled trial comparing radiation doses of 50.4 vs. $64.8 \mathrm{~Gy}$, with concurrent CF, no survival benefit was achieved with the increased radiation dose (5). Therefore, in the US and Europe, CF in combination with a radiation dose of 50.4 Gy is commonly used for EC, as in the RTOG 85-01 study. However, $\mathrm{CF}$ is associated with renal and gastrointestinal toxicity, thrombosis, and other AEs linked to cisplatin, and hospital admission is required for continuous 5-FU infusion.

FOLFOX is one of the global standard treatments for colorectal cancer $(16,17)$. In the US and Europe, oxaliplatin may be used for treatment of EC (2). FOLFOX is expected to contribute to a reduction in toxicity and to increase outpatient treatment through replacement of cisplatin with oxaliplatin in CRT for EC. The results of phase I and II clinical studies of CRT with FOLFOX for EC have been promising (18-20). Conroy et al conducted phase I and II clinical trials of FOLFOX with CRT for EC and obtained favorable results (7). Consequently, randomized phase II and III studies comparing FOLFOX with CF have been conducted, including PRODIGE5/ACCORD17 (8). The results of the phase II study were promising but, unexpectedly, the primary endpoint was not met in the phase III study, as FOLFOX was not found to be superior in terms of PFS with statistical significance [9.7 months for the FOLFOX group vs. 9.4 months for the CF group; hazard ratio $(\mathrm{HR})=0.93 ; 95 \%$ confidence interval (CI): 0.7-1.24; $\mathrm{P}=0.64)$. However, due to the lower rates of nephrotoxicity, treatment-related death/sudden death, and other toxicities, and due to the fact that it can be administered on an outpatient basis, CRT with FOLFOX is commonly used in clinical practice in western countries.

In Japan and worldwide, cisplatin has been replaced with oxaliplatin for gastric cancer treatment, as it reduces gastrointestinal toxicity and enables outpatient treatment (3,21-24). However, the use of oxaliplatin for EC has not been approved. Therefore, clinical data of FOLFOX for EC in Japan were available only from patients with multiple primary cancers, for which oxaliplatin is approved. Watanabe et al administered CRT with FOLFOX to a patient who had both locally advanced ESCC and synchronous metastatic colon cancer (25). Unlike the ACCORD17 study, an $80 \%$ dose of 5-FU-oxaliplatin-leucovorin (mFOLFOX6) (26), which is commonly used for colorectal cancer, was used. Although a tracheoesophageal fistula developed at the end of treatment, the patient was able to complete CRT with FOLFOX, and the tumor size was reduced. The only other grade 3 adverse event was leukocytopenia (25). However, such cases are usually excluded from clinical trials, and little is known on the feasibility of this treatment for Japanese EC patients.

The 4 patients in the present study had both ESCC and advanced RC. As patients 3 and 4 were able to ingest food and did not require hydration, they were treated as outpatients and they achieved a CR for ESCC. Moreover, patient 3 also achieved a CR for RC and was able to avoid surgery. Patient 4 underwent CRT with FOLFOX as postoperative adjuvant 
chemotherapy for RC and achieved a satisfactory progression-free period. Although neither patient 1 nor patient 2 achieved a CR for ESCC, the primary lesions were reduced in size, and progression of RC was arrested. In other words, both ESCC and RC were well controlled, and the patients were able to complete CRT with FOLFOX for ESCC. These experiences suggest that CRT with FOLFOX may be a useful therapeutic option for such patients, at least in Japan.

We should be careful regarding the extrapolation of this regimen to ESCC in our country due to the differences in clinical practice between Japan and other countries. First, the major histological type of EC is adenocarcinoma in western countries and SCC in Asia. However, although evidence of this regimen has been verified in US and Europe, several trials include a non-negligible ESCC population. Chiarion et al reported that their study included $85 \%$ cases of ESCC, and PRODIGE5/ACCORD17 consists of $85-86 \%$ of cases of ESCC $(8,19)$. Therefore, CRT with FOLFOX for ESCC may be acceptable. Another point is the difference in the radiation dose. Although a radiation dose of 50.4 Gy was used in an overseas phase III clinical study (5), a dose of $60 \mathrm{~Gy} / 30 \mathrm{Fr}$ is the standard in Japan $(10,11)$. Following a discussion, we decided to use full-dose radiation as the domestic standard in cases 1,3 and 4, as salvage surgery was unlikely, in an attempt to avoid non-CR. Despite the high-dose radiation, neither acute nor late toxicity was observed. Thus, $50.4 \mathrm{~Gy}$ will be adopted as it is estimated to be an acceptable dose for ESCC in Japan.

Patient 2 was scheduled to receive neoadjuvant CRT; therefore, an exposure dose of 41.4 Gy was used, and he also did not experience any clinically significant toxicity. He developed an AE of grade $\geq 3$ (5-FU-induced encephalitis), but this toxicity was predictable and it was quickly treated as reported in the literature (27).

Unlike CRT with CF, CRT with FOLFOX did not cause any specific or serious AEs in the 4 reported cases. Although attention should be paid to variations in chemotherapy cycles or irradiation dose and fields in each case, which is the limitation of retrospective case studies, the safety and efficacy of radiation with 3 cycles of FOLFOX may be discussed in approximation.

In conclusion, the present study suggests that CRT with FOLFOX may be well tolerated and feasible in patients with ESCC and synchronous RC, although the retrospective nature of the study and the small number of patients in Japan constitute major limitations. As the antitumor activity of this treatment was found to be highly promising, further investigation with more subjects is required.

\section{Acknowledgements}

Not applicable.

\section{Funding}

No funding was received.

\section{Availability of data and materials}

The datasets used and/or analyzed during the present study are available from the corresponding author on reasonable request.

\section{Authors' contributions}

TY, HH, MA, YK, SM, NT, TM and SS and YS contributed to the treatment and follow-up examinations, and TY wrote the manuscript. All the authors have read and approved the final version of this manuscript for publication.

\section{Ethics approval and consent to participate}

The present study was approved by the Ethics Committee of Saitama Cancer Center.

\section{Patient consent for publication}

The requirement for the patients' written consent was waived with removal of all identifying information in this retrospective study under the approval of the Ethics Committee of Saitama Cancer Center.

\section{Competing interests}

Hiroki Hara received grants from the following companies: Astrazeneca, Daiichi Sankyo, Dainippon Sumitomo Pharma, Lilly, Merck Serono, MSD, Taiho, Chugai, Eisai, LSK BioPharma, Incyte, Pfizer, Boehringer-Ingelheim, Beigene, ONO and BMS. These grants did not directly support this submitted study. All the other authors declare that they have no competing interests to disclose.

\section{References}

1. Global Burden of Disease Cancer Collaboration, Fitzmaurice C, Dicker D, Pain A, Hamavid H, Moradi-Lakeh M, MacIntyre MF, Allen C, Hansen G, Woodbrook R, et al: The global burden of cancer 2013. JAMA Oncol 1: 505-527, 2015.

2. National Comprehensive Cancer Network: Clinical practice oncology: Esophageal and esophagogastric junction cancers (version 2. 2017).

3. Stahl M, Mariette C, Haustermans K, Cervantes A and Arnold D; ESMO Guidelines Working Group: Oesophageal cancer: ESMO clinical practice guidelines for diagnosis, treatment and follow-up. Ann Oncol 24 (Suppl 6): vi51-vi56, 2013.

4. Cooper JS, Guo MD, Herskovic A, Macdonald JS, Martenson JA Jr, Al-Sarraf M, Byhardt R, Russell AH, Beitler JJ, Spencer S, et al: Chemoradiotherapy of locally advanced esophageal cancer: Long-term follow-up of a prospective randomized trial (RTOG 85-01). Radiation therapy oncology group. JAMA 281: 1623-1627, 1999.

5. Minsky BD, Pajak TF, Ginsberg RJ, Pisansky TM, Martenson J, Komaki R, Okawara G, Rosenthal SA and Kelsen DP: INT 0123 (radiation therapy oncology group 94-05) phase III trial of combined-modality therapy for esophageal cancer: High-dose versus standard-dose radiation therapy. J Clin Oncol 20: 1167-1174, 2002.

6. al-Sarraf M, Martz K, Herskovic A, Leichman L, Brindle JS Vaitkevicius VK, Cooper J, Byhardt R, Davis L and Emami B: Progress report of combined chemoradiotherapy versus radiotherapy alone in patients with esophageal cancer: An intergroup study. J Clin Oncol 15: 277-284, 1997.

7. Conroy T, Yataghene Y, Etienne PL, Michel P, Senellart H, Raoul JL, Mineur L, Rives M, Mirabel X, Lamezec B, et al: Phase II randomised trial of chemoradiotherapy with FOLFOX4 or cisplatin plus fluorouracil in oesophageal cancer. $\mathrm{Br}$ J Cancer 103: 1349-1355, 2010.

8. Conroy T, Galais MP, Raoul JL, Bouche O, Gourgou-Bourgade S, Douillard JY, Etienne PL, Boige V, Martel-Lafay I, Michel P, et al: Definitive chemoradiotherapy with FOLFOX versus fluorouracil and cisplatin in patients with oesophageal cancer (PRODIGE5/ACCORD17): Final results of a randomised, phase 2/3 trial. Lancet Oncol 15: 305-314, 2014. 
9. Messager M, Mirabel X, Tresch E, Paumier A, Vendrely V, Dahan L, Glehen O, Vasseur F, Lacornerie T, Piessen G, et al: Preoperative chemoradiation with paclitaxel-carboplatin or with fluorouracil-oxaliplatin-folinic acid (FOLFOX) for resectable esophageal and junctional cancer: The PROTECT-1402, randomized phase 2 trial. BMC Cancer 16: 318, 2016

10. Shinoda M, Ando N, Kato K, Ishikura S, Kato H, Tsubosa Y, Minashi K, Okabe H, Kimura Y, Kawano T, et al: Randomized study of low-dose versus standard-dose chemoradiotherapy for unresectable esophageal squamous cell carcinoma (JCOG0303). Cancer Sci 106: 407-412, 2015.

11. Kato K, Muro K, Minashi K, Ohtsu A, Ishikura S, Boku N, Takiuchi H, Komatsu Y, Miyata Y, Fukuda H, et al: Phase II study of chemoradiotherapy with 5-fluorouracil and cisplatin for stage II-III esophageal squamous cell carcinoma: JCOG trial (JCOG 9906). Int J Radiat Oncol Biol Phys 81: 684-690, 2011.

12. Kato H, Sato A, Fukuda H, Kagami Y, Udagawa H, Togo A, Ando N, Tanaka O, Shinoda M, Yamana H and Ishikura S: A phase II trial of chemoradiotherapy for stage I esophageal squamous cell carcinoma: Japan clinical oncology group study (JCOG9708). Jpn J Clin Oncol 39: 638-643, 2009.

13. Japan Esophageal Society: Japanase classification of esophageal cancer, 11th edition: Part II and III. Esophagus 14: 37-65, 2017.

14. Eisenhauera EA, Therasseb P, Bogaertsc J, Schwartzd H, Sargente D, Ford R,, Dancey J, Gwyther S, Mooney M, Rubinstein L, et al: New response evaluation criteria in solid tumours: Revised RECIST guideline (version 1.1). Eur J Cancer 45: 228-247, 2009.

15. National Institutes of Health Common Terminology Criteria for Adverse Events (CTCAE) Version 4.0. US Department of Health and Human Services, National Institutes of Health, National Cancer Center, National Cancer Center, Washington DC, 2009.

16. de Gramont A, Figer A, Seymour M, Homerin M, Hmissi A, Cassidy J, Boni C, Cortes-Funes H, Cervantes A, Freyer G, et al: Leucovorin and fluorouracil with or without oxaliplatin as first-line treatment in advanced colorectal cancer. J Clin Oncol 18: 2938-2947, 2000.

17. Andre T, Boni C, Mounedji-Boudiaf L, Navarro M, Tabernero J, Hickish T, Topham C, Zaninelli M, Clingan P, Bridgewater J, et al: Oxaliplatin, fluorouracil, and leucovorin as adjuvant treatment for colon cancer. N Engl J Med 350: 2343-2351, 2004.

18. Khushalani NI, Leichman CG, Proulx G, Nava H, Bodnar L, Klippenstein D, Litwin A, Smith J, Nava E, Pendyala L, et al Oxaliplatin in combination with protracted-infusion fluorouracil and radiation: Report of a clinical trial for patients with esophageal cancer. J Clin Oncol 20: 2844-2850, 2002.
19. Chiarion-Sileni V, Innocente R, Cavina R, Ruol A, Corti L, Pigozzo J, Del Bianco P, Fumagalli U, Santoro A and Ancona E: Multi-center phase II trial of chemo-radiotherapy with 5-fluorouracil, leucovorin and oxaliplatin in locally advanced esophageal cancer. Cancer Chemother Pharmacol 63: 1111-1119, 2009.

20. O'Connor BM, Chadha MK, Pande A, Lombardo JC, Nwogu CE, Nava HR, Yang G and Javle MM: Concurrent oxaliplatin, 5-fluorouracil, and radiotherapy in the treatment of locally advanced esophageal carcinoma. Cancer J 13: 119-124, 2007.

21. Cunningham D, Allum WH, Stenning SP, Thompson JN, Van de Velde CJ, Nicolson M, Scarffe JH, Lofts FJ, Falk SJ, Iveson TJ, et al: Perioperative chemotherapy versus surgery alone for resectable gastroesophageal cancer. N Engl J Med 355: 11-20, 2006.

22. Bang YJ, Kim YW, Yang HK, Chung HC, Park YK, Lee KH, Lee KW, Kim YH, Noh SI, Cho JY, et al: Adjuvant capecitabine and oxaliplatin for gastric cancer after D2 gastrectomy (CLASSIC): A phase 3 open-label, randomised controlled trial. Lancet 379: 315-321, 2012.

23. Yamada Y, Higuchi K, Nishikawa K, Gotoh M, Fuse N, Sugimoto N, Nishina T, Amagai K, Chin K, Niwa Y, et al: Phase III study comparing oxaliplatin plus S-1 with cisplatin plus $\mathrm{S}-1$ in chemotherapy-naive patients with advanced gastric cancer. Ann Oncol 26: 141-148, 2015.

24. Masuishi T, Kadowaki S, Kondo M, Komori A, Sugiyama K, Mitani S, Honda K, Narita Y, Taniguchi H, Ura T, et al: FOLFOX as first-line therapy for gastric cancer with severe peritoneal metastasis. Anticancer Res 37: 7037-7042, 2017.

25. Watanabe Y, Tsutsui M, Takeda S, Yoshino S and Oka M: A case of esophageal cancer associated with colon cancer successfully treated with combination chemotherapy of FOLFOX and concurrent radiotherapy. Gan To Kagaku Ryoho 36: 2439-2441, 2009 (In Japanese).

26. Allegra CJ, Yothers G, O'Connell MJ, Sharif S, Colangelo LH, Lopa SH, Petrelli NJ, Goldberg RM, Atkins JN, Seay TE, et al: Initial safety report of NSABP C-08: A randomized phase III study of modified FOLFOX6 with or without bevacizumab for the adjuvant treatment of patients with stage II or III colon cancer. J Clin Oncol 27: 3385-3390, 2009.

27. Mitani S, Kadowaki S, Kato K, Masuishi T and Muro K: Combination of oxaliplatin and 5-fluorouracil/leucovorin for advanced esophageal squamous cell carcinoma refractory or intolerant to standard therapies. Case Rep Oncol 12: 304-310, 2019.

This work is licensed under a Creative Commons Attribution-NonCommercial-NoDerivatives 4.0 International (CC BY-NC-ND 4.0) License. 